\title{
Resolution of Fuzzy Relational Inequalities with Boolean Semi-Tensor Product Composition
}

\author{
Shuling Wang and Haitao Li *(1) \\ School of Mathematics and Statistics, Shandong Normal University, Jinan 250014, China; \\ 2018020496@stu.sdnu.edu.cn \\ * Correspondence: lihaitao@sdnu.edu.cn or haitaoli09@gmail.com
}

check for updates

Citation: Wang, S.; Li, H. Resolution of Fuzzy Relational Inequalities with Boolean Semi-Tensor Product Composition. Mathematics 2021, 9 , 937. https://doi.org/10.3390/ math9090937

Academic Editors: Michael Voskoglou and Salvatore Sessa

Received: 14 March 2021

Accepted: 21 April 2021

Published: 23 April 2021

Publisher's Note: MDPI stays neutral with regard to jurisdictional claims in published maps and institutional affiliations.

Copyright: (c) 2021 by the authors. Licensee MDPI, Basel, Switzerland. This article is an open access article distributed under the terms and conditions of the Creative Commons Attribution (CC BY) license (https:// creativecommons.org/licenses/by/ $4.0 /)$.

\begin{abstract}
Resolution of fuzzy relational inequalities (FRIs) plays a significant role in decision-making, image compression and fuzzy control. This paper studies the resolution of a kind of FRIs with Boolean semi-tensor product composition. First, by resorting to the column stacking technique, the equivalent form of FRIs with Boolean semi-tensor product composition is obtained, which is a system of FRIs (SFRIs) with max-min composition. Second, based on the semi-tensor product method, all the solutions to FRIs with Boolean semi-tensor product composition are obtained by finding all possible parameter set solutions. Finally, a general procedure is developed for the resolution of FRIs with Boolean semi-tensor product composition. Two illustrative examples are worked out to show the effectiveness of the obtained new results.
\end{abstract}

Keywords: fuzzy relational inequality; Boolean semi-tensor product composition; column stacking; semi-tensor product of matrices

\section{Introduction}

Resolution of fuzzy relational equations (FREs) and fuzzy relational inequalities (FRIs) has wide applications in several research fields including decision-making, image compression, fuzzy control and so on [1-4]. E. Sanchez initiated the resolution theory of FREs and applied it to medical research [5]. Since then, the resolution of FREs (FRIs) has become a heated topic [6-8]. The resolution of FREs with max-product composition was considered in [9-11]. Cornejo et al. [12,13] investigated the solvability of bipolar max-product FREs. Several effective alternatives for solving fuzzy nonlinear equations were proposed in [14-16]. An algorithm for solving FREs with max-T composition was established in [17]. The resolution of FREs with max-min composition was investigated in [18-20]. Besides the study of FREs and FRIs, the resolution of system of fuzzy relational equations (SFREs) and system of fuzzy relational inequalities (SFRIs) has also been widely studied $[21,22]$.

Recently, the semi-tensor product of matrices has been put forward by Cheng [23], which has been widely applied to the analysis and control of finite-value dynamical systems, including controllability [24,25], observability [26], stability and stabilization [27-29], optimal control [30], synchronization [31], game theory [32-36] and so on [37,38]. Lu et al. [39] presented a detailed survey on the applications of semi-tensor product of matrices to finitevalue dynamical systems. In particular, the semi-tensor product method has also been applied to the modeling of fuzzy systems [40,41] and resolution of FREs and FRIs [42,43]. Cheng et al. [44] first applied the semi-tensor product method to the resolution of FREs with max-min composition. Based on the semi-tensor product of matrices, Li and Wang [45] studied the resolution of FRIs with max-min composition. Several kinds of FRIs and SFRIs with max-min composition were considered in [46] by virtue of a column stacking approach.

In the past decade, dimension-varying systems have received intensive attention due to the wide applications in spacecrafts, vehicle clutch systems and biological systems [47]. When considering dimension-varying fuzzy systems, it is significant to deal with fuzzy 
relations with incompatible dimensions. However, all the existing results on the resolution of FREs and FRIs just considered the case where fuzzy matrices have compatible dimensions (see Definition 2 below). When the dimensions of two fuzzy matrices are not compatible, we call it Boolean semi-tensor product composition. Therefore, it is meaningful to investigate the resolution of FREs and FRIs with Boolean semi-tensor product composition, and apply the obtained results to the study of dimension-varying fuzzy systems. It is easy to see that the max-min composition is a special case of Boolean semi-tensor product composition.

This paper focuses on the resolution of FRIs (see (11) below) and SFRIs (see (12) below) with Boolean semi-tensor product composition, and aims to propose a general procedure to obtain all the solutions. The main contributions of this paper are two-fold. On one hand, we investigate the basic theory of Boolean semi-tensor product. Compared with semi-tensor product, we find that some important properties of semi-tensor product such as associative law, pseudo commutativity, and the properties of swap matrix and transpose operator still hold for Boolean semi-tensor product. On the other hand, we establish a general procedure for the resolution of FRIs and SFRIs with Boolean semi-tensor product composition, which facilitates the application of fuzzy theory in dimension-varying systems.

The remainder of this paper is organized as follows. In Section 2, we recall some necessary preliminaries. Section 3 formulates the problems studied in this paper and studies the equivalent forms of FRIs and SFRIs with Boolean semi-tensor product composition. In Section 4, a general procedure is established for the resolution of the considered FRIs and SFRIs. Two numerical examples are given to support our new results in Section 5, which is followed by a brief conclusion in Section 6 .

\section{Preliminaries}

\subsection{Semi-Tensor Product of Matrices}

In this part, we present some necessary preliminaries on the semi-tensor product of matrices. For details, please refer to [23,44].

Definition 1. Let $P \in \mathcal{M}_{m \times n}, Q \in \mathcal{M}_{s \times t}$. Denote the least common multiple of $n$ and $s$ by $\alpha=\operatorname{lcm}(n, s)$. Then, the semi-tensor product of $P$ and $Q$ is

$$
P \ltimes Q=\left(P \otimes I_{\frac{\alpha}{n}}\right)\left(Q \otimes I_{\frac{\alpha}{s}}\right),
$$

where $\otimes$ is the Kronecker product.

Lemma 1. Let $X \in \mathcal{M}_{s \times 1}$ be a column vector and $P \in \mathcal{M}_{m \times n}$. Then

$$
X \ltimes P=\left(I_{s} \otimes P\right) \ltimes X .
$$

Denote $\mathcal{D}_{k}:=\left\{0, \frac{1}{k-1}, \cdots, \frac{k-2}{k-1}, 1\right\}$. When $k=\infty, \mathcal{D}_{\infty}:=[0,1]$. Define $\Delta_{s}:=\left\{\delta_{s}^{i}: i=\right.$ $1,2, \cdots, s\}$, where $\delta_{s}^{i}$ denotes the $i$-th column of identity matrix $I_{s}$. Identify $\frac{i}{k-1}$ as $\delta_{k}^{k-i}$, $i=0,1, \cdots, k-1$. Then, one can see that $\mathcal{D}_{k} \sim \Delta_{k} . \delta_{k}^{k-i}$ is called the vector form of $\frac{i}{k-1}$ and we do not distinguish $\delta_{k}^{k-i}$ and $\frac{i}{k-1}$ if no confusion arises in the sequel. For any $a, b \in \mathcal{D}_{k}$, $a \vee_{k} b=\max \{a, b\}, a \wedge_{k} b=\min \{a, b\}$. When $k=\infty, a \vee b:=a \vee_{\infty} b$ and $a \wedge b:=a \wedge_{\infty} b$.

Lemma 2. Let $x, y \in \mathcal{D}_{k}$. Then

(i)

$$
x \vee_{k} y=M_{d}^{k} \ltimes x \ltimes y,
$$

(ii)

$$
\text { where } M_{d}^{k}=\delta_{k}\left[P_{1} P_{2} \cdots P_{k}\right] \text {, and } P_{r}=\left[\begin{array}{lllr}
1 & 2 \cdots r & \cdots & \underbrace{r \cdots r}_{k-r+1}
\end{array}\right], r=1,2, \cdots, k \text {; }
$$

$$
x \wedge_{k} y=M_{c}^{k} \ltimes x \ltimes y,
$$


where $M_{c}^{k}=\delta_{k}\left[Q_{1} Q_{2} \cdots Q_{k}\right]$, and $Q_{r}=[\underbrace{r \cdots r}_{r} r+1 r+2 \cdots k], r=1,2, \cdots, k$.

\subsection{Boolean Semi-Tensor Product Composition}

To formulate the problem considered in this paper, we introduce some necessary operators. Denote the set of $s \times t$ matrices with their entries in $\mathcal{D}_{k}$ by $\mathcal{D}_{k}^{s \times t}$.

Definition 2. Let $P=\left(p_{i, j}\right) \in \mathcal{D}_{\infty}^{m \times n}, Q=\left(q_{i, j}\right) \in \mathcal{D}_{\infty}^{n \times s}$. Then, the max-min composition operator, denoted by " $\circ$ ", is defined as

$$
R=\left(r_{i, j}\right)=P \circ Q \in \mathcal{D}_{\infty}^{m \times s},
$$

where $r_{i, j}=\vee_{k=1}^{n}\left(p_{i, k} \wedge q_{k, j}\right)$.

Definition 3. Let $P=\left(p_{i, j}\right) \in \mathcal{D}_{\infty}^{m \times n}, Q=\left(q_{i, j}\right) \in \mathcal{D}_{\infty}^{s \times t}$. Then, the Boolean Kronecker product of $P$ and $Q$, denoted by $P \otimes_{\mathcal{B}} Q \in \mathcal{D}_{\infty}^{m s \times n t}$, is

$$
P \otimes_{\mathcal{B}} Q=\left[\begin{array}{ccc}
p_{1,1} \otimes_{\mathcal{B}} Q & \cdots & p_{1, n} \otimes_{\mathcal{B}} Q \\
\vdots & & \vdots \\
p_{m, 1} \otimes_{\mathcal{B}} Q & \cdots & p_{m, n} \otimes_{\mathcal{B}} Q
\end{array}\right],
$$

where

$$
p_{i, j} \otimes_{\mathcal{B}} Q=\left[\begin{array}{ccc}
p_{i, j} \wedge q_{1,1} & \cdots & p_{i, j} \wedge q_{1, t} \\
\vdots & & \vdots \\
p_{i, j} \wedge q_{s, 1} & \cdots & p_{i, j} \wedge q_{s, t}
\end{array}\right]
$$

Similar to the ordinary Kronecker product, one can obtain the following properties of Boolean Kronecker product.

Proposition 1. (i) Let $P, Q, R$ be three real matrices with arbitrary dimensions. Then

$$
P \otimes_{\mathcal{B}} Q \otimes_{\mathcal{B}} R=P \otimes_{\mathcal{B}}\left(Q \otimes_{\mathcal{B}} R\right) .
$$

(ii) Let $P \in \mathcal{M}_{m \times n}, Q \in \mathcal{M}_{s \times t}$. Then

$$
\left(P \otimes_{\mathcal{B}} Q\right)^{\top}=P^{\top} \otimes_{\mathcal{B}} Q^{\top} .
$$

Definition 4. Let $P=\left(p_{i, j}\right) \in \mathcal{D}_{\infty}^{m \times n}, Q=\left(q_{i, j}\right) \in \mathcal{D}_{\infty}^{s \times t}$. Then, the Boolean semi-tensor product composition operator, denoted by " $\ltimes_{\mathcal{B}}$ ", is defined as

$$
P \ltimes_{\mathcal{B}} Q=\left(P \otimes_{\mathcal{B}} I_{\frac{\alpha}{n}}\right) \circ\left(Q \otimes_{\mathcal{B}} I_{\frac{\alpha}{\mathcal{S}}}\right),
$$

where $\alpha=\operatorname{lcm}(n, s)$.

Remark 1. One can easily see from Definition 4 that $P \ltimes_{\mathcal{B}} Q=P \circ Q$ holds for $n=s$.

Next, we present some important properties of Boolean semi-tensor product composition.

Proposition 2. Let $P, Q, R$ be three real matrices with arbitrary dimensions. Then

$$
P \ltimes_{\mathcal{B}} Q \ltimes_{\mathcal{B}} R=P \ltimes_{\mathcal{B}}\left(Q \ltimes_{\mathcal{B}} R\right) .
$$

Proposition 3. Let $P \in \mathcal{M}_{m \times n}, Q \in \mathcal{M}_{s \times t}$. Then

$$
\left(P \ltimes_{\mathcal{B}} Q\right)^{\top}=Q^{\top} \ltimes_{\mathcal{B}} P^{\top} .
$$


Proof of Proposition 3. A simple calculation shows that

$$
\begin{aligned}
\left(P \ltimes_{\mathcal{B}} Q\right)^{\top} & =\left[\left(P \otimes_{\mathcal{B}} I_{\frac{\alpha}{n}}\right) \circ\left(Q \otimes_{\mathcal{B}} I_{\frac{\alpha}{s}}\right)\right]^{\top} \\
& =\left(Q \otimes_{\mathcal{B}} I_{\frac{\alpha}{s}}\right)^{\top} \circ\left(P \otimes_{\mathcal{B}} I_{\frac{\alpha}{n}}\right)^{\top} \\
& =\left(Q^{\top} \otimes_{\mathcal{B}} I_{\frac{\alpha}{s}}\right) \circ\left(P^{\top} \otimes_{\mathcal{B}} I_{\frac{\alpha}{n}}\right) \\
& =Q^{\top} \ltimes_{\mathcal{B}} P^{\top},
\end{aligned}
$$

where $\alpha=\operatorname{lcm}(n, s)$.

Proposition 4. Let $X \in \mathcal{D}_{\infty}^{s \times 1}$ and $Y \in \mathcal{D}_{\infty}^{t \times 1}$ be two column vectors. Then

$$
W_{[s, t]} \ltimes_{\mathcal{B}} X \ltimes_{\mathcal{B}} Y=Y \ltimes_{\mathcal{B}} X,
$$

where $W_{[s, t]}:=\left[\begin{array}{llllll}I_{t} \otimes \delta_{s}^{1} & I_{t} \otimes \delta_{s}^{2} & \cdots & I_{t} \otimes \delta_{s}^{s}\end{array}\right]$.

Proof of Proposition 4. Set $X=\left[\begin{array}{llll}x_{1} & x_{2} & \cdots & x_{s}\end{array}\right]^{\top}$ and $Y=\left[\begin{array}{llll}y_{1} & y_{2} & \cdots & y_{t}\end{array}\right]^{\top}$. Then, it holds that

$$
\begin{aligned}
X \ltimes_{\mathcal{B}} Y= & \left(X \otimes_{\mathcal{B}} I_{t}\right) \circ Y \\
= & {\left[x_{1} \wedge y_{1} x_{1} \wedge y_{2} \cdots x_{1} \wedge y_{t} x_{2} \wedge y_{1} x_{2} \wedge y_{2} \cdots x_{2} \wedge y_{t}\right.} \\
& \left.\cdots x_{s} \wedge y_{1} x_{s} \wedge y_{2} \cdots x_{s} \wedge y_{t}\right]^{\top} .
\end{aligned}
$$

Thus,

$$
\begin{aligned}
W_{[s, t]} \ltimes_{\mathcal{B}} X \ltimes_{\mathcal{B}} Y= & W_{[s, t]} \ltimes_{\mathcal{B}}\left(X \ltimes_{\mathcal{B}} Y\right) \\
= & {\left[x_{1} \wedge y_{1} x_{2} \wedge y_{1} \cdots x_{s} \wedge y_{1} x_{1} \wedge y_{2} x_{2} \wedge y_{2} \cdots x_{s} \wedge y_{2}\right.} \\
& \left.\cdots x_{1} \wedge y_{t} x_{2} \wedge y_{t} \cdots x_{s} \wedge y_{t}\right]^{\top} \\
= & Y \ltimes_{\mathcal{B}} X .
\end{aligned}
$$

\section{Problem Formulation}

In this paper, we consider the following two problems:

- $\quad$ Problem 1: Solve the following FRI:

$$
G \leqslant A \ltimes_{\mathcal{B}} X \leqslant H,
$$

where $X \in \mathcal{D}_{\infty}^{p \times q}$ is an unknown matrix, $A \in \mathcal{D}_{\infty}^{m \times n}, G, H \in \mathcal{D}_{\infty}^{\frac{\alpha m}{n} \times \frac{\alpha q}{p}}$ are known matrices, and $\alpha=\operatorname{lcm}(n, p)$.

- Problem 2: Solve the following SFRIs:

$$
\left\{\begin{array}{c}
G_{1} \leq A_{1} \ltimes_{\mathcal{B}} X \leq H_{1}, \\
G_{2} \leq A_{2} \ltimes_{\mathcal{B}} X \leq H_{2}, \\
\vdots \\
G_{N} \leq A_{N} \ltimes_{\mathcal{B}} X \leq H_{N},
\end{array}\right.
$$

where $X \in \mathcal{D}_{\infty}^{p \times q}$ is an unknown matrix, $A_{i} \in \mathcal{D}_{\infty}^{m_{i} \times n_{i}}, G_{i}, H_{i} \in \mathcal{D}_{\infty}^{\frac{\alpha_{i} m_{i}}{n_{i}} \times \frac{\alpha_{i} q}{p}}$ are known matrices, $\alpha_{i}=\operatorname{lcm}\left(n_{i}, p\right), i=1,2, \cdots, N$, and $N \in \mathbb{Z}_{+}, N \geq 2$.

Let $A=\left(a_{i, j}\right) \in \mathcal{M}_{s \times t}$. Then, the column stacking form of $A$, denoted by $V_{c}(A) \in \mathcal{M}_{s t \times 1}$, is defined as

$$
V_{c}(A)=\left[a_{1,1} a_{2,1} \cdots a_{s, 1} \cdots a_{1, t} a_{2, t} \cdots a_{s, t}\right]^{\top} .
$$


Using column stacking operator, we present the equivalent forms of FRI (11) and SFRIs (12) successively.

Proposition 5. Let $A \in \mathcal{D}_{\infty}^{m \times n}, B \in \mathcal{D}_{\infty}^{n \times p}$. Then

$$
V_{c}(A \circ B)=\left(I_{p} \otimes_{\mathcal{B}} A\right) \circ V_{c}(B) .
$$

Proof of Proposition 5. A direct calculation shows that

$$
\begin{aligned}
V_{c}(A \circ B) & =V_{c}\left(\left[A \circ \operatorname{Col}_{1}(B) A \circ \operatorname{Col}_{2}(B) \cdots A \circ \operatorname{Col}_{p}(B)\right]\right) \\
& =\left[\begin{array}{c}
A \circ \operatorname{Col}_{1}(B) \\
A \circ \operatorname{Col}_{2}(B) \\
\vdots \\
A \circ \operatorname{Col}_{p}(B)
\end{array}\right]=\left[\begin{array}{cccc}
A & \mathbf{0} & \cdots & \mathbf{0} \\
\mathbf{0} & A & \cdots & \mathbf{0} \\
\vdots & & & \vdots \\
\mathbf{0} & \mathbf{0} & \cdots & A
\end{array}\right] \circ\left[\begin{array}{c}
\operatorname{Col}_{1}(B) \\
\operatorname{Col}_{2}(B) \\
\vdots \\
\operatorname{Col}_{p}(B)
\end{array}\right] \\
= & \left(I_{p} \otimes_{\mathcal{B}} A\right) \circ V_{c}(B),
\end{aligned}
$$

where $\operatorname{Col}_{i}(B)$ denotes the $i$-th column of $B, i=1,2, \cdots, p$.

Proposition 6. Let $A \in \mathcal{D}_{\infty}^{p \times q}$. Then

$$
V_{c}\left(A \otimes_{\mathcal{B}} I_{s}\right)=T_{p, q}^{s} \circ V_{c}(A),
$$

where

$$
T_{p, q}^{\mathcal{S}}=W_{[s, q]} \ltimes_{\mathcal{B}} W_{[p q, s]} \ltimes_{\mathcal{B}} W_{\left[s^{2}, p q\right]} \ltimes_{\mathcal{B}}\left(V_{\mathcal{c}}\left(I_{S}\right) \otimes_{\mathcal{B}} I_{p q}\right) .
$$

Proof of Proposition 6. Let

$$
\begin{aligned}
\xi_{i}^{j} & =\left[a_{1, i} \wedge\left(\delta_{s}^{j}\right)^{\top} a_{2, i} \wedge\left(\delta_{s}^{j}\right)^{\top} \cdots a_{p, i} \wedge\left(\delta_{s}^{j}\right)^{\top}\right], i=1,2, \cdots, q, j=1,2, \cdots, s ; \\
\zeta_{i, j} & =\left[a_{i, j} \wedge\left(\delta_{s}^{1}\right)^{\top} a_{i, j} \wedge\left(\delta_{s}^{2}\right)^{\top} \cdots a_{i, j} \wedge\left(\delta_{s}^{s}\right)^{\top}\right], i=1,2, \cdots, p, j=1,2, \cdots, q .
\end{aligned}
$$

By Definition 4, it is easy to obtain that

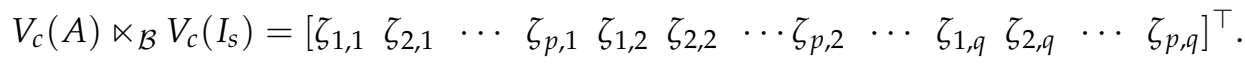

Then, we have

$$
\begin{aligned}
& W_{[s, q]} \ltimes_{\mathcal{B}} W_{[p q, s]} \ltimes_{\mathcal{B}} V_{\mathcal{c}}(A) \ltimes_{\mathcal{B}} V_{\mathcal{C}}\left(I_{s}\right) \\
& =W_{[s, q]} \ltimes_{\mathcal{B}}\left[\begin{array}{lllllllllllll}
\xi_{1}^{1} & \xi_{2}^{1} & \cdots & \xi_{q}^{1} & \xi_{1}^{2} & \xi_{2}^{2} & \cdots & \xi_{q}^{2} & \cdots & \xi_{1}^{s} & \xi_{2}^{s} & \cdots & \xi_{q}^{s}
\end{array}\right]^{\top} \\
& =\left[\begin{array}{lllllllllllll}
\xi_{1}^{1} & \xi_{1}^{2} & \cdots & \xi_{1}^{s} & \xi_{2}^{1} & \xi_{2}^{2} & \cdots & \xi_{2}^{s} & \cdots & \xi_{q}^{1} & \xi_{q}^{2} & \cdots & \xi_{q}^{s}
\end{array}\right]^{\top} \\
& =V_{c}\left(A \otimes_{\mathcal{B}} I_{S}\right) \text {, }
\end{aligned}
$$

which together with (8) and (10) shows that

$$
\begin{aligned}
V_{c}\left(A \otimes_{\mathcal{B}} I_{S}\right) & =W_{[s, q]} \ltimes_{\mathcal{B}} W_{[p q, s]} \ltimes_{\mathcal{B}} V_{c}(A) \ltimes_{\mathcal{B}} V_{c}\left(I_{s}\right) \\
& =W_{[s, q]} \ltimes_{\mathcal{B}} W_{[p q, s]} \ltimes_{\mathcal{B}} W_{\left[s^{2}, p q\right]} \ltimes_{\mathcal{B}} V_{c}\left(I_{S}\right) \ltimes_{\mathcal{B}} V_{c}(A) \\
& =\left[W_{[s, q]} \ltimes_{\mathcal{B}} W_{[p q, s]} \ltimes_{\mathcal{B}} W_{\left[s^{2}, p q\right]} \ltimes_{\mathcal{B}}\left(V_{c}\left(I_{S}\right) \otimes_{\mathcal{B}} I_{p q}\right)\right] \circ V_{c}(A) \\
& =T_{p, q}^{s} \circ V_{\mathcal{C}}(A) .
\end{aligned}
$$


Remark 2. Precisely, for (14), we have

$$
\begin{aligned}
& T_{p, q}^{\mathcal{S}}=W_{[s, q]} \ltimes_{\mathcal{B}} W_{[p q, s]} \ltimes_{\mathcal{B}} W_{\left[s^{2}, p q\right]} \ltimes_{\mathcal{B}}\left(V_{c}\left(I_{s}\right) \otimes_{\mathcal{B}} I_{p q}\right) \\
& =[\underbrace{\delta_{p q}^{1} \mathbf{0} \cdots \mathbf{0}}_{s} \cdots \underbrace{\delta_{p q}^{p} \mathbf{0} \cdots \mathbf{0}}_{s} \underbrace{\mathbf{0} \delta_{p q}^{1} \mathbf{0} \cdots \mathbf{0}}_{s} \cdots \underbrace{\mathbf{0} \delta_{p q}^{p} \mathbf{0} \cdots \mathbf{0}}_{s} \cdots \underbrace{\mathbf{0} \cdots \mathbf{0} \delta_{p q}^{1}}_{s} \cdots \\
& \underbrace{\mathbf{0} \cdots \mathbf{0} \delta_{p q}^{p}}_{s} \underbrace{\delta_{p q}^{p+1} \mathbf{0} \cdots \mathbf{0}}_{s} \cdots \underbrace{\delta_{p q}^{2 p} \mathbf{0} \cdots \mathbf{0}}_{s} \underbrace{\mathbf{0} \delta_{p q}^{p+1} \mathbf{0} \cdots \mathbf{0}}_{s} \cdots \underbrace{\mathbf{0} \delta_{p q}^{2 p} \mathbf{0} \cdots \mathbf{0}}_{s} \cdots \\
& \underbrace{\mathbf{0} \cdots \mathbf{0} \delta_{p q}^{p+1}}_{s} \cdots \underbrace{\mathbf{0} \cdots \mathbf{0} \delta_{p q}^{2 p}}_{s} \cdots \underbrace{\delta_{p q}^{(q-1) p+1} \mathbf{0} \cdots \mathbf{0}}_{s} \cdots \underbrace{\delta_{p q}^{q p} \mathbf{0} \cdots \mathbf{0}}_{s} \\
& \underbrace{\mathbf{0} \delta_{p q}^{(q-1) p+1} \mathbf{0} \cdots \mathbf{0}}_{s} \cdots \underbrace{\mathbf{0} \delta_{p q}^{q p} \mathbf{0} \cdots \mathbf{0}}_{s} \cdots \underbrace{\mathbf{0} \cdots \mathbf{0} \delta_{p q}^{(q-1) p+1}}_{s} \cdots
\end{aligned}
$$

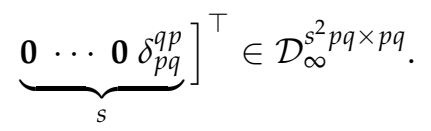

Proposition 7. Let $A \in \mathcal{D}_{\infty}^{m \times n}, B \in \mathcal{D}_{\infty}^{p \times q}$. Then

$$
V_{c}\left(A \ltimes_{\mathcal{B}} B\right)=K \circ V_{c}(B),
$$

where $K=\left(I_{\frac{\alpha q}{p}} \otimes_{\mathcal{B}} A \otimes_{\mathcal{B}} I_{\frac{\alpha}{n}}\right) \circ T_{p, q}^{\frac{\alpha}{p}} \in \mathcal{D}_{\infty}^{\frac{\alpha^{2} m q}{n p} \times p q}$ and $\alpha=\operatorname{lcm}(n, p)$.

Proof of Proposition 7. By Definition 4, Propositions 5 and 6, one can obtain that

$$
\begin{aligned}
V_{c}\left(A \ltimes_{\mathcal{B}} B\right) & =V_{c}\left(\left(A \otimes_{\mathcal{B}} I_{\frac{\alpha}{n}}\right) \circ\left(B \otimes_{\mathcal{B}} I_{\frac{\alpha}{p}}\right)\right) \\
& =\left[I_{\frac{\alpha q}{p}} \otimes_{\mathcal{B}}\left(A \otimes_{\mathcal{B}} I_{\frac{\alpha}{n}}\right)\right] \circ V_{\mathcal{c}}\left(B \otimes_{\mathcal{B}} I_{\frac{\alpha}{p}}\right) \\
& =\left(I_{\frac{\alpha q}{p}} \otimes_{\mathcal{B}} A \otimes_{\mathcal{B}} I_{\frac{\alpha}{n}}\right) \circ\left(T_{p, q}^{\frac{\alpha}{p}} \circ V_{\mathcal{c}}(B)\right) \\
& =\left[\left(I_{\frac{\alpha q}{p}} \otimes_{\mathcal{B}} A \otimes_{\mathcal{B}} I_{\frac{\alpha}{n}}\right) \circ T_{p, q}^{\frac{\alpha}{p}}\right] \circ V_{c}(B) .
\end{aligned}
$$

Remark 3. The matrix $\mathrm{K}$ in (15) can be represented as the following block matrix:

$$
K=\left[\begin{array}{cccc}
K^{\prime} & \mathbf{0} & \cdots & \mathbf{0} \\
\mathbf{0} & K^{\prime} & \cdots & \mathbf{0} \\
\vdots & \vdots & & \vdots \\
\mathbf{0} & \mathbf{0} & \cdots & K^{\prime}
\end{array}\right]
$$

where $K^{\prime}=\delta_{q}^{1^{\top}} \ltimes_{\mathcal{B}}\left[\left(I_{\frac{\alpha q}{p}} \otimes_{\mathcal{B}} A \otimes_{\mathcal{B}} I_{\frac{\alpha}{n}}\right) \circ T_{p, q}^{\frac{\alpha}{p}}\right] \ltimes_{\mathcal{B}} \delta_{q}^{1} \in \mathcal{D}_{\infty}^{\frac{\alpha^{2} m}{n p} \times p}$.

Next, we present an example to illustrate Proposition 7.

Example 1. Given $A=\left[\begin{array}{cccc}0.1 & 0.5 & 0.7 & 0.2 \\ 1 & 0.3 & 0.2 & 0.6 \\ 0 & 0.2 & 0.4 & 0.3\end{array}\right] \in \mathcal{D}_{\infty}^{3 \times 4}$, and assume that $X=\left(x_{i, j}\right) \in$ $\mathcal{D}_{\infty}^{2 \times 3}$ is an unknown matrix. 
By Proposition 7, we have

$$
V_{c}\left(A \ltimes_{\mathcal{B}} X\right)=\left[\left(I_{6} \otimes_{\mathcal{B}} A\right) \circ T_{2,3}^{2}\right] \circ V_{\mathcal{c}}(X),
$$

where

$$
I_{6} \otimes_{\mathcal{B}} A=\left[\begin{array}{ccc}
A & \cdots & \mathbf{0} \\
\vdots & & \vdots \\
\mathbf{0} & \cdots & A
\end{array}\right] \in \mathcal{D}_{\infty}^{18 \times 24}
$$

and $T_{2,3}^{2}=\left[\begin{array}{llllllllllllllllllllllll}\delta_{6}^{1} & \mathbf{0} & \delta_{6}^{2} & \mathbf{0} & \mathbf{0} & \delta_{6}^{1} & \mathbf{0} & \delta_{6}^{2} & \delta_{6}^{3} & \mathbf{0} & \delta_{6}^{4} & \mathbf{0} & \mathbf{0} & \delta_{6}^{3} & \mathbf{0} & \delta_{6}^{4} & \delta_{6}^{5} & \mathbf{0} & \delta_{6}^{6} & \mathbf{0} & \mathbf{0} & \delta_{6}^{5} & \mathbf{0} & \delta_{6}^{6}\end{array}\right]^{\top}$. Thus,

$$
V_{c}\left(A \ltimes_{\mathcal{B}} X\right)=K \circ\left[\begin{array}{llllll}
x_{1,1} & x_{2,1} & x_{1,2} & x_{2,2} & x_{1,3} & x_{2,3}
\end{array}\right]^{\top}
$$

where $K=\left[\begin{array}{ccc}K^{\prime} & \mathbf{0} & \mathbf{0} \\ \mathbf{0} & K^{\prime} & \mathbf{0} \\ \mathbf{0} & \mathbf{0} & K^{\prime}\end{array}\right]$, and $K^{\prime}=\left[\begin{array}{cccccc}0.1 & 0.6 & 0.6 & 1 & 0.3 & 0.1 \\ 0.6 & 1 & 0.1 & 0.6 & 0.1 & 0.3\end{array}\right]^{\top}$.

Based on Proposition 7 and Remark 3, we have the following equivalent form of FRI (11).

Theorem 1. FRI (11) is equivalent to the following SFRIs composed of FRIs with the max-min composition:

$$
\left\{\begin{array}{c}
\mathbf{g}^{1} \leq K^{\prime} \circ \mathbf{x}^{1} \leq \mathbf{h}^{1} \\
\mathbf{g}^{2} \leq K^{\prime} \circ \mathbf{x}^{2} \leq \mathbf{h}^{2} \\
\vdots \\
\mathbf{g}^{q} \leq K^{\prime} \circ \mathbf{x}^{q} \leq \mathbf{h}^{q}
\end{array}\right.
$$

where

$$
\begin{aligned}
& \mathbf{g}=V_{c}(G)=\left[\left(\mathbf{g}^{1}\right)^{\top}\left(\mathbf{g}^{2}\right)^{\top} \cdots\left(\mathbf{g}^{q}\right)^{\top}\right]^{\top} \in \mathcal{D}_{\infty}^{\frac{\alpha^{2} m q}{n p} \times 1}, \mathbf{g}^{j} \in \mathcal{D}_{\infty}^{\frac{\alpha^{2} m}{n p} \times 1}, j=1,2, \cdots, q ; \\
& \mathbf{h}=V_{c}(H)=\left[\left(\mathbf{h}^{1}\right)^{\top}\left(\mathbf{h}^{2}\right)^{\top} \cdots\left(\mathbf{h}^{q}\right)^{\top}\right]^{\top} \in \mathcal{D}_{\infty}^{\frac{\alpha^{2} m q}{n p} \times 1}, \mathbf{h}^{j} \in \mathcal{D}_{\infty}^{\frac{\alpha^{2} m}{n p} \times 1}, j=1,2, \cdots, q ; \\
& \mathbf{x}=V_{c}(X)=\left[\left(\mathbf{x}^{1}\right)^{\top}\left(\mathbf{x}^{2}\right)^{\top} \cdots\left(\mathbf{x}^{q}\right)^{\top}\right]^{\top} \in \mathcal{D}_{\infty}^{p q \times 1}, \mathbf{x}^{j} \in \mathcal{D}_{\infty}^{p \times 1}, j=1,2, \cdots, q ;
\end{aligned}
$$

and

$$
\begin{aligned}
& K=\left(I_{\frac{\alpha q}{p}} \otimes_{\mathcal{B}} A \otimes_{\mathcal{B}} I_{\frac{\alpha}{n}}\right) \circ T_{p, q}^{\frac{\alpha}{p}}=\left[\begin{array}{cccc}
K^{\prime} & \mathbf{0} & \cdots & \mathbf{0} \\
\mathbf{0} & K^{\prime} & \cdots & \mathbf{0} \\
\vdots & \vdots & & \vdots \\
\mathbf{0} & \mathbf{0} & \cdots & K^{\prime}
\end{array}\right] \in \mathcal{D}_{\infty}^{\frac{\alpha^{2} m q}{n p} \times p q}, \\
& K^{\prime}=\delta_{q}^{1^{\top}} \ltimes_{\mathcal{B}}\left[\left(I_{\frac{\alpha q}{p}} \otimes_{\mathcal{B}} A \otimes_{\mathcal{B}} I_{\frac{\alpha}{n}}\right) \circ T_{p, q}^{\frac{\alpha}{p}}\right] \ltimes_{\mathcal{B}} \delta_{q}^{1} \in \mathcal{D}_{\infty}^{\frac{\alpha^{2} m}{n p} \times p} .
\end{aligned}
$$

Proof of Proposition 1. The proof of this theorem is based on a straightforward calculation, and thus we omit it here.

Example 2. Recall Example 1 and given

$$
G=\left[\begin{array}{cccccc}
0.1 & 0.2 & 0.4 & 0.3 & 0.2 & 0.3 \\
0.2 & 0.1 & 0.3 & 0.1 & 0 & 0.2 \\
0 & 0.1 & 0.2 & 0.2 & 0 & 0.2
\end{array}\right], H=\left[\begin{array}{cccccc}
0.3 & 0.5 & 1 & 0.5 & 0.4 & 0.5 \\
0.4 & 0.4 & 0.6 & 0.3 & 0.4 & 0.5 \\
0.3 & 0.2 & 0.5 & 0.3 & 0.3 & 1
\end{array}\right]
$$

Then, according to Theorem 1, FRI

$$
G \leq A \ltimes_{\mathcal{B}} X \leq H
$$


is equivalent to the following SFRIs composed of FRIs with the max-min composition:

$$
\left\{\begin{array}{l}
\mathbf{g}^{1} \leq K^{\prime} \circ \mathbf{x}^{1} \leq \mathbf{h}^{1} \\
\mathbf{g}^{2} \leq K^{\prime} \circ \mathbf{x}^{2} \leq \mathbf{h}^{2} \\
\mathbf{g}^{3} \leq K^{\prime} \circ \mathbf{x}^{3} \leq \mathbf{h}^{3}
\end{array},\right.
$$

where $\mathbf{x}^{i} \in \mathcal{D}_{\infty}^{2 \times 1}, \mathbf{g}^{i}, \mathbf{h}^{i} \in \mathcal{D}_{\infty}^{6 \times 1}, i=1,2,3$ satisfy $V_{c}(X)=\left[\left(\mathbf{x}^{1}\right)^{\top}\left(\mathbf{x}^{2}\right)^{\top}\left(\mathbf{x}^{3}\right)^{\top}\right]^{\top}$, $V_{c}(G)=\left[\left(\mathbf{g}^{1}\right)^{\top}\left(\mathbf{g}^{2}\right)^{\top}\left(\mathbf{g}^{3}\right)^{\top}\right]^{\top}$ and $V_{c}(H)=\left[\left(\mathbf{h}^{1}\right)^{\top}\left(\mathbf{h}^{2}\right)^{\top}\left(\mathbf{h}^{3}\right)^{\top}\right]^{\top}$, respectively, and $K^{\prime}$ is given in Example 1.

Similar to Theorem 1, we can also present the equivalent form of SFRIs (12).

Theorem 2. SFRIs (12) is equivalent to the following SFRIs composed of FRIs with the max-min composition:

$$
\left\{\begin{array}{c}
\mathbf{g}^{1,1} \leq K^{1^{\prime}} \circ \mathbf{x}^{1} \leq \mathbf{h}^{1,1} \\
\mathbf{g}^{1,2} \leq K^{1^{\prime}} \circ \mathbf{x}^{2} \leq \mathbf{h}^{1,2} \\
\vdots \\
\mathbf{g}^{1, q} \leq K^{1^{\prime}} \circ \mathbf{x}^{q} \leq \mathbf{h}^{1, q}, \\
\vdots \\
\mathbf{g}^{N, 1} \leq K^{N^{\prime}} \circ \mathbf{x}^{1} \leq \mathbf{h}^{N, 1}, \\
\mathbf{g}^{N, 2} \leq K^{N^{\prime}} \circ \mathbf{x}^{2} \leq \mathbf{h}^{N, 2} \\
\quad \vdots \\
\mathbf{g}^{N, q} \leq K^{N^{\prime}} \circ \mathbf{x}^{q} \leq \mathbf{h}^{N, q},
\end{array}\right.
$$

where

$$
\begin{aligned}
& \mathbf{g}^{i}=V_{\mathcal{c}}\left(G_{i}\right)=\left[\left(\mathbf{g}^{i, 1}\right)^{\top}\left(\mathbf{g}^{i, 2}\right)^{\top} \cdots\left(\mathbf{g}^{i, q}\right)^{\top}\right]^{\top} \in \mathcal{D}_{\infty}^{\frac{\alpha_{i}^{2} m_{i} q}{n_{i} p} \times 1}, \mathbf{g}^{i, j} \in \mathcal{D}_{\infty}^{\frac{\alpha_{i}^{2} m_{i}}{n_{i} p} \times 1} ; \\
& \mathbf{h}^{i}=V_{\mathcal{c}}\left(H_{i}\right)=\left[\left(\mathbf{h}^{i, 1}\right)^{\top}\left(\mathbf{h}^{i, 2}\right)^{\top} \cdots\left(\mathbf{h}^{i, q}\right)^{\top}\right]^{\top} \in \mathcal{D}_{\infty}^{\frac{\alpha_{i}^{2} m_{i} q}{n_{i} p} \times 1}, \mathbf{h}^{i, j} \in \mathcal{D}_{\infty}^{\frac{\alpha_{i}^{2} m_{i}}{n_{i} p} \times 1} ; \\
& \mathbf{x}=V_{c}(X)=\left[\left(\mathbf{x}^{1}\right)^{\top}\left(\mathbf{x}^{2}\right)^{\top} \cdots\left(\mathbf{x}^{q}\right)^{\top}\right]^{\top} \in \mathcal{D}_{\infty}^{p q \times 1}, \mathbf{x}^{j} \in \mathcal{D}_{\infty}^{p \times 1} ; \\
& K^{i}=\left(I_{\frac{\alpha_{i} q}{p}} \otimes_{\mathcal{B}} A_{i} \otimes_{\mathcal{B}} I_{\frac{\alpha_{i}}{n_{i}}}\right) \circ T_{p, q}^{\frac{\alpha_{i}}{p}}=\left[\begin{array}{cccc}
K^{i^{\prime}} & \mathbf{0} & \cdots & \mathbf{0} \\
\mathbf{0} & K^{i^{\prime}} & \cdots & \mathbf{0} \\
\vdots & \vdots & & \vdots \\
\mathbf{0} & \mathbf{0} & \cdots & K^{i^{\prime}}
\end{array}\right] \in \mathcal{D}_{\infty}^{\frac{\alpha_{i}^{2} m_{i} q}{n_{i} p} \times p q}, \\
& K^{i^{\prime}}=\delta_{q}^{1^{\top}} \ltimes_{\mathcal{B}}\left[\left(I_{\frac{\alpha_{i} q}{p}} \otimes_{\mathcal{B}} A_{i} \otimes_{\mathcal{B}} I_{\frac{\alpha_{i}}{n_{i}}}\right) \circ T_{p, q}^{\frac{\alpha_{i}}{p}}\right] \ltimes_{\mathcal{B}} \delta_{q}^{1} \in \mathcal{D}_{\infty}^{\frac{\alpha_{i}^{2} m_{i}}{n_{i} p} \times p}, \\
& \text { and } i=1,2, \cdots, N, j=1,2, \cdots, q \text {. }
\end{aligned}
$$

Example 3. Recall Examples 1 and 2. Given

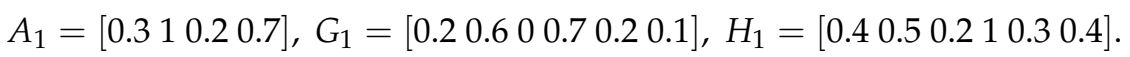

Then, according to Theorem 2, SFRI

$$
\left\{\begin{array}{l}
G \leq A \ltimes_{\mathcal{B}} X \leq H, \\
G_{1} \leq A_{1} \ltimes_{\mathcal{B}} X \leq H_{1}
\end{array}\right.
$$


is equivalent to the following SFRIs composed of FRIs with the max-min composition:

$$
\left\{\begin{array}{l}
\mathbf{g}^{1} \leq K^{\prime} \circ \mathbf{x}^{1} \leq \mathbf{h}^{1} \\
\mathbf{g}^{2} \leq K^{\prime} \circ \mathbf{x}^{2} \leq \mathbf{h}^{2}, \\
\mathbf{g}^{3} \leq K^{\prime} \circ \mathbf{x}^{3} \leq \mathbf{h}^{3} \\
\mathbf{g}^{1,1} \leq K^{1^{\prime}} \circ \mathbf{x}^{1} \leq \mathbf{h}^{1,1} \\
\mathbf{g}^{1,2} \leq K^{1^{\prime}} \circ \mathbf{x}^{2} \leq \mathbf{h}^{1,2} \\
\mathbf{g}^{1,3} \leq K^{1^{\prime}} \circ \mathbf{x}^{3} \leq \mathbf{h}^{1,3},
\end{array}\right.
$$

where $K^{1^{\prime}}=\left[\begin{array}{cc}0.3 & 0.2 \\ 1 & 0.7\end{array}\right], \mathbf{g}^{1, i}, \mathbf{h}^{1, i} \in \mathcal{D}_{\infty}^{2 \times 1}, i=1,2,3$ satisfy $V_{c}\left(G_{1}\right)=\left[\left(\mathbf{g}^{1,1}\right)^{\top}\left(\mathbf{g}^{1,2}\right)^{\top}\right.$ $\left.\left(\mathbf{g}^{1,3}\right)^{\top}\right]^{\top}$ and $V_{c}\left(H_{1}\right)=\left[\left(\mathbf{h}^{1,1}\right)^{\top}\left(\mathbf{h}^{1,2}\right)^{\top}\left(\mathbf{h}^{1,3}\right)^{\top}\right]^{\top}$, respectively, $K^{\prime}$ is given in Example 1, and $\mathbf{x}^{i}, \mathbf{g}^{i}, \mathbf{h}^{i}, i=1,2,3$ are given in Example 2.

Remark 4. From Theorems 1 and 2, one can see that both FRI (11) and SFRIs (12) are equivalent to SFRIs composed of FRIs with the max-min composition. Thus, the column stacking technique unifies the resolution of (11) and (12), and converts Problems 1 and 2 into the resolution of FRIs with the max-min composition. Therefore, one just needs to study the resolution of the following FRI with the max-min composition:

$$
\mathbf{u} \leq W \circ \mathbf{x} \leq \mathbf{v},
$$

where $\mathbf{u}=\left[\begin{array}{llll}u_{1} & u_{2} & \cdots & u_{m}\end{array}\right]^{\top}, \mathbf{v}=\left[\begin{array}{llll}v_{1} & v_{2} & \cdots & v_{m}\end{array}\right]^{\top} \in \mathcal{D}_{\infty}^{m \times 1}, W=\left(w_{i, j}\right) \in \mathcal{D}_{\infty}^{m \times n}$ and $\mathbf{x}=\left[\begin{array}{llll}x_{1} & x_{2} & \cdots & x_{n}\end{array}\right]^{\top} \in \mathcal{D}_{\infty}^{n \times 1}$.

\section{Resolution of FRI (20)}

In this section, we investigate the resolution of FRI (11) and SFRIs (12) via solving FRI (20). To this end, we recall some results on the resolution of FRI (20). For details, please refer to $[42,44,45]$.

Definition 5. Denote the solution set of FRI (20) by $X(W, \mathbf{u}, \mathbf{v}) \subseteq \mathcal{D}_{\infty}^{n \times 1}$.

(i) If $\overline{\mathbf{x}} \geq \mathbf{x}$ holds for any $\mathbf{x} \in X(W, \mathbf{u}, \mathbf{v})$, then $\overline{\mathbf{x}} \in X(W, \mathbf{u}, \mathbf{v})$ is called the maximum solution;

(ii) If for any $\mathbf{x} \in X(W, \mathbf{u}, \mathbf{v}), \mathbf{x} \leq \underline{\mathbf{x}}$ implies $\mathbf{x}=\underline{\mathbf{x}}$, then $\underline{\mathbf{x}} \in X(W, \mathbf{u}, \mathbf{v})$ is called a minimal solution.

Denote the solution set of FRI (20) by $\Omega$. It was pointed out in [45] that the solution set of FRIs with max-min composition can be characterized by the unique maximum solution and finite minimal solutions. More specifically, it holds that

$$
\Omega=\bigcup_{i=1}^{s}\left[\begin{array}{c}
\underline{x}_{1}^{i} \leq x_{1} \leq \bar{x}_{1} \\
\underline{x}_{2}^{i} \leq x_{2} \leq \bar{x}_{2} \\
\vdots \\
\underline{x}_{n}^{i} \leq x_{n} \leq \bar{x}_{n}
\end{array}\right],
$$

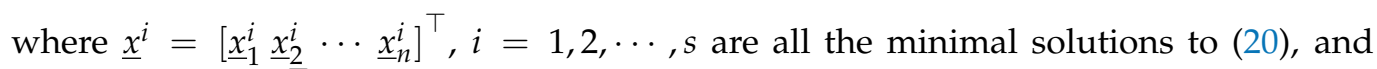
$\bar{x}=\left[\begin{array}{lll}\bar{x}_{1} \bar{x}_{2} & \cdots & \bar{x}_{n}\end{array}\right]^{\top}$ is the unique maximum solution to (20). In addition, it shows that all the minimal solutions and the unique maximum solution are within the set of parameter set solutions. Keeping these points in mind, we calculate the set of parameter set solutions to FRI (20) based on semi-tensor product of matrices. 
Denote the parameter set of FRI (20) by $\Phi=\left\{\varphi_{1}, \varphi_{2}, \cdots, \varphi_{l}\right\}$, and identify $\varphi_{i} \sim \frac{i-1}{l-1} \sim$ $\delta_{l}^{l-i+1}, \varphi_{i} \in \Phi, i=1,2, \cdots, l$. Then, we have $\Phi \sim \mathcal{D}_{l} \sim \Delta_{l}$. We say $\delta_{l}^{l-i_{1}+1} \leq \delta_{l}^{l-i_{2}+1}$, if $\varphi_{i_{1}} \geq \varphi_{i_{2}}$.

Then, for the $i$-th inequality of (20), i.e.,

$$
u_{i} \leq\left(w_{i, 1} \wedge x_{1}\right) \vee\left(w_{i, 2} \wedge x_{2}\right) \vee \cdots \vee\left(w_{i, n} \wedge x_{n}\right) \leq v_{i}
$$

by Lemma 1, we can convert the middle part of (22) into the following form:

$$
\begin{aligned}
& \left(w_{i, 1} \wedge x_{1}\right) \vee\left(w_{i, 2} \wedge x_{2}\right) \vee \cdots \vee\left(w_{i, n} \wedge x_{n}\right) \\
= & \left(M_{d}^{l}\right)^{n-1} \ltimes_{t=1}^{n}\left[I_{l^{t-1}} \otimes\left(M_{c}^{l} \ltimes w_{i, t}\right)\right] \ltimes_{j=1}^{n} x_{j} \\
= & N_{i} \ltimes x,
\end{aligned}
$$

where $N_{i}=\left(M_{d}^{l}\right)^{n-1} \ltimes_{t=1}^{n}\left[I_{l^{t-1}} \otimes\left(M_{c}^{l} \ltimes w_{i, t}\right)\right], x=\ltimes_{j=1}^{n} x_{j}$, and $M_{d}^{l}, M_{c}^{l}$ are given in Lemma 2. Then, (22) becomes

$$
u_{i} \geq N_{i} \ltimes x \geq v_{i},
$$

where $N_{i}$ is a logical matrix, $x \in \Delta_{l n}$ and $u_{i}, v_{i} \in \Delta_{l}$.

Noting that $N_{i} \ltimes \delta_{l^{n}}^{k}=\operatorname{Col}_{k}\left(N_{i}\right)$, then we can obtain the following result.

Lemma 3. $\mathbf{x}=\left[\begin{array}{llll}x_{1} & x_{2} & \cdots & x_{n}\end{array}\right]^{\top} \in \mathcal{D}_{\infty}^{n \times 1}$ with $x=\ltimes_{j=1}^{n} x_{j}=\delta_{l^{n}}^{k}$ is a parameter set solution to FRI (22), if and only if

$$
u_{i} \geq \operatorname{Col}_{k}\left(N_{i}\right) \geq v_{i}
$$

The following proposition is crucial for the resolution of FRI (22), which can be obtained by Lemma 3.

Lemma 4. Assume that $K_{i}=\left\{k: u_{i} \geq \operatorname{Col}_{k}\left(N_{i}\right) \geq v_{i}\right\}, i=1,2, \cdots, m$. Then, the set of parameter set solutions to FRI (22), denoted by $\Lambda_{i}$, is

$$
\Lambda_{i}=\left\{\mathbf{x}=\left[\begin{array}{llll}
x_{1} & x_{2} & \cdots & x_{n}
\end{array}\right]^{\top} \in \mathcal{D}_{\infty}^{n \times 1} \text { with } \ltimes_{j=1}^{n} x_{j}=\delta_{l^{n}}^{k}: k \in K_{i}\right\} .
$$

Thus, the set of parameter set solutions to (20) is

$$
\Lambda=\cap_{i=1}^{m} \Lambda_{i}
$$

Moreover, if $\Lambda=\varnothing$, then FRI (20) has no solution.

To sum up, we have the following procedure (Table 1) on the resolution of FRI (11) and SFRIs (12). Figure 1 presents the flowchart of the procedure given in Table 1.

Remark 5. Since $A \ltimes_{\mathcal{B}} X \geq G$ and $A \ltimes_{\mathcal{B}} X \leq H$ can be converted into $G \leq A \ltimes_{\mathcal{B}} X \leq \mathbf{1}$ and $\mathbf{0} \leq A \ltimes_{\mathcal{B}} X \leq H$, respectively, Table 1 can also be applied to the resolution of these two kinds of FRIs. When $G=H, F R I$ (11) becomes an FRE. Thus, Table 1 can be used to solve FRE $A \ltimes_{\mathcal{B}} X=G$. 
Table 1. Procedure on the resolution of FRI (11) and SFRIs (12).

To obtain all the solutions to FRI (11) (resp. SFRIs (12)), one can proceed by the following steps:

(1) Calculate the equivalent form of FRI (11) (resp. SFRIs (12)) by Theorem 1 (resp. Theorem 2);

(2) Construct the parameter set $\Phi^{j}$ of the $j$-th FRI in SFRIs (16) (resp. (19)), and give the vector form of every element in $\Phi^{j}$;

(3) Calculate $\Lambda^{j}$ by Lemma 4;

(4) Obtain all the minimal solutions and the unique maximum solution to the $j$-th FRI in (16) (resp. (19)) by comparing the finite number of elements in $\Lambda^{j}$;

(5) Obtain the solution set $\Omega^{j}$ of the $j$-th FRI in (16) (resp. (19)) by (21);

(6) Obtain the solution set $\Omega$ of FRI (11) (resp. SFRIs (12)).

Remark 6. One can convert $\tilde{G} \leq X \ltimes_{\mathcal{B}} \tilde{A} \leq \tilde{H}$ into $\tilde{G}^{\top} \leq \tilde{A}^{\top} \ltimes_{\mathcal{B}} X^{\top} \leq \tilde{H}^{\top}$ by (9), which has the same form with FRI (11). Therefore, Table 1 is also applicable to finding all the solutions to this kind of FRI.

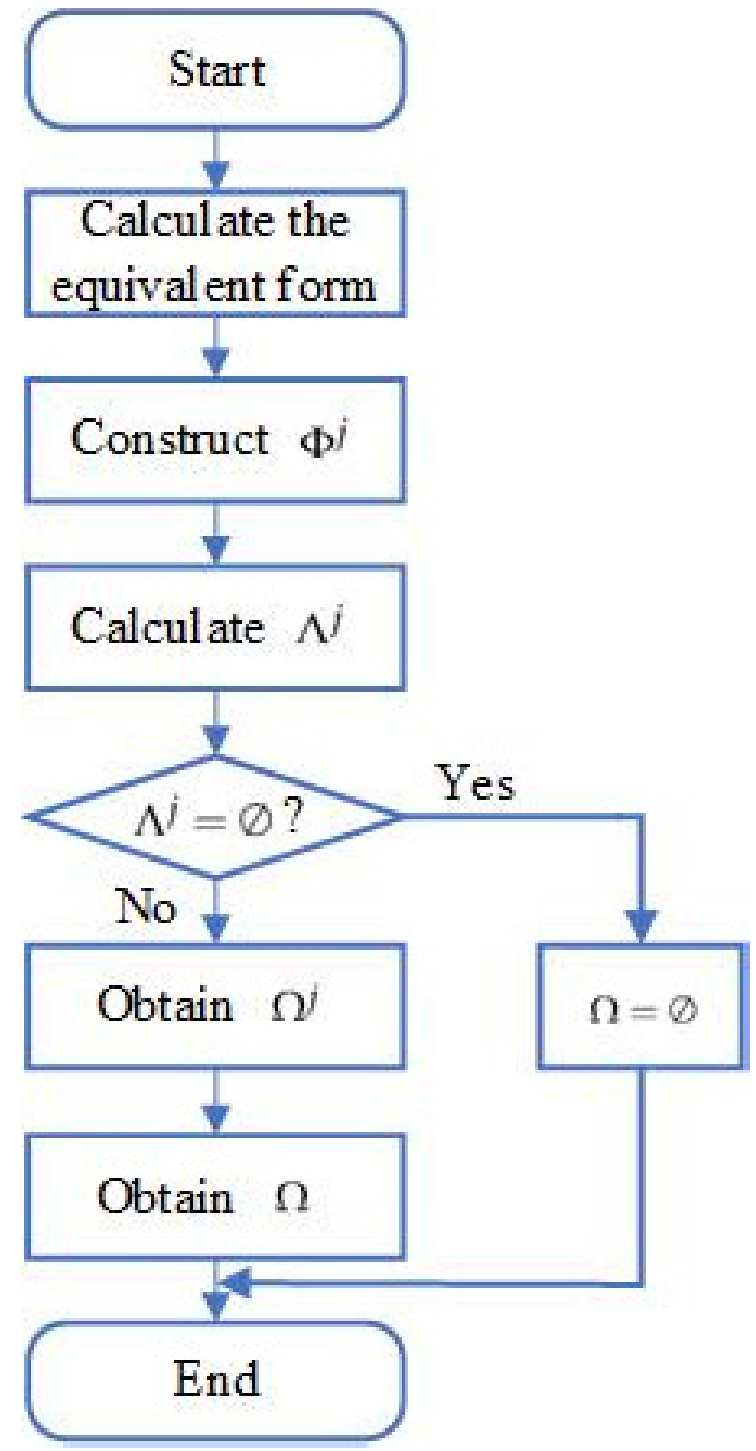

Figure 1. Flowchart of obtaining all the solutions to FRI (11) (resp. SFRIs (12)). 


\section{Illustrative Examples}

In this section, we give two examples to illustrate the main results.

Example 4. Recall Example 2 and consider the resolution of FRI (17).

In Example 2, we have obtained the equivalent form of FRI (17), i.e., SFRIs (18). In the following, we solve every FRI in (18).

To obtain the solution set of the first FRI in (18), we first find the following parameter set:

$$
\Phi^{1}=\{0,0.1,0.2,0.3,0.4,0.5,0.6,1\},
$$

and identify each element of $\Phi^{1}$ by vector form as

$$
0 \sim \delta_{8}^{8}, 0.1 \sim \delta_{8}^{7}, 0.2 \sim \delta_{8}^{6}, 0.3 \sim \delta_{8}^{5}, 0.4 \sim \delta_{8}^{4}, 0.5 \sim \delta_{8}^{3}, 0.6 \sim \delta_{8}^{2}, 1 \sim \delta_{8}^{1} .
$$

Next, we calculate all the parameter set solutions to the first FRI in (18).

For the following first inequality of the first FRI in (18):

$$
0.1 \leq\left(0.1 \wedge x_{1,1}\right) \vee\left(0.6 \wedge x_{2,1}\right) \leq 0.3,
$$

by Lemmas 1 and 2, we obtain its algebraic form as follows:

$$
\delta_{8}^{7} \geq M^{1,1} \ltimes x^{1} \geq \delta_{8}^{5},
$$

where

$$
M^{1,1}=M_{d}^{8} \ltimes_{t=1}^{2}\left[I_{8^{t-1}} \otimes\left(M_{c}^{8} \ltimes k_{1, t}^{\prime}\right)\right]=\delta_{8}[22345677 \cdots 22345678]
$$

and $x^{1}=x_{1,1} \ltimes x_{2,1}$. Then, according to Lemma 4 , the set of parameter set solutions to the first inequality of the first FRI in (18) is

$$
\begin{aligned}
\Lambda^{1,1}= & \left\{\delta_{64}^{5}, \cdots, \delta_{64}^{8}, \delta_{64}^{13}, \cdots, \delta_{64}^{16}, \delta_{64}^{21}, \cdots, \delta_{64}^{24}, \delta_{64}^{29}, \cdots, \delta_{64}^{32},\right. \\
& \left.\delta_{64}^{37}, \cdots, \delta_{64}^{40}, \delta_{64}^{45}, \cdots, \delta_{64}^{48}, \delta_{64}^{53}, \cdots, \delta_{64}^{56}, \delta_{64}^{61}, \cdots, \delta_{64}^{63}\right\} .
\end{aligned}
$$

Similarly, we can respectively convert other inequalities of the first FRI in (18) into the following algebraic forms:

$$
\begin{gathered}
\delta_{8}^{6} \geq M^{1,2} \ltimes x^{1} \geq \delta_{8}^{4}, \delta_{8}^{8} \geq M^{1,3} \ltimes x^{1} \geq \delta_{8}^{5}, \\
\delta_{8}^{6} \geq M^{1,4} \ltimes x^{1} \geq \delta_{8}^{3}, \delta_{8}^{7} \geq M^{1,5} \ltimes x^{1} \geq \delta_{8}^{4}, \\
\delta_{8}^{7} \geq M^{1,6} \ltimes x^{1} \geq \delta_{8}^{6},
\end{gathered}
$$

where

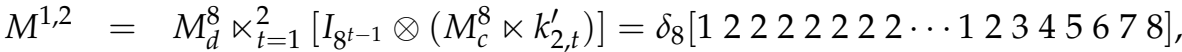

$$
\begin{aligned}
& M^{1,3}=M_{d}^{8} \ltimes_{t=1}^{2}\left[I_{8^{t-1}} \otimes\left(M_{c}^{8} \ltimes k_{3, t}^{\prime}\right)\right]=\delta_{8}[222222222 \cdots 77777778],
\end{aligned}
$$

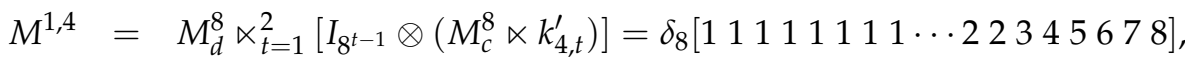

$$
\begin{aligned}
& M^{1,5}=M_{d}^{8} \ltimes_{t=1}^{2}\left[I_{8^{t-1}} \otimes\left(M_{c}^{8} \ltimes k_{5, t}^{\prime}\right)\right]=\delta_{8}[55555555 \cdots 77777778] \text {, } \\
& M^{1,6}=M_{d}^{8} \ltimes_{t=1}^{2}\left[I_{8^{t-1}} \otimes\left(M_{c}^{8} \ltimes k_{6, t}^{\prime}\right)\right]=\delta_{8}[55555677 \cdots 55555678] \text {. }
\end{aligned}
$$


Then, the corresponding set of parameter set solutions to each inequality is

$$
\begin{aligned}
\Lambda^{1,2}= & \left\{\delta_{64}^{28}, \cdots, \delta_{64}^{32}, \delta_{64}^{36}, \cdots, \delta_{64}^{40}, \delta_{64}^{44}, \cdots, \delta_{64}^{48}, \delta_{64}^{52}, \cdots, \delta_{64}^{54}, \delta_{64}^{60}, \cdots, \delta_{64}^{62}\right\}, \\
\Lambda^{1,3}= & \left\{\delta_{64}^{33}, \delta_{64}^{34} \cdots, \delta_{64}^{64}\right\}, \\
\Lambda^{1,4}= & \left\{\delta_{64}^{19}, \cdots, \delta_{64}^{24}, \delta_{64}^{27}, \cdots, \delta_{64}^{32}, \delta_{64}^{35}, \cdots, \delta_{64}^{40}, \delta_{64}^{43}, \cdots, \delta_{64}^{48}, \delta_{64}^{51}, \cdots, \delta_{64}^{54},\right. \\
& \left.\delta_{64}^{58} \cdots, \delta_{64}^{62}\right\}, \\
\Lambda^{1,5}= & \left\{\delta_{64}^{1}, \delta_{64}^{2} \cdots, \delta_{64}^{63}\right\}, \\
\Lambda^{1,6}= & \left\{\delta_{64}^{6}, \cdots, \delta_{64}^{8}, \delta_{64}^{14}, \cdots, \delta_{64}^{16}, \delta_{64}^{22}, \cdots, \delta_{64}^{24}, \delta_{64}^{30}, \cdots, \delta_{64}^{32}, \delta_{64}^{38}, \cdots, \delta_{64}^{40},\right. \\
& \left.\delta_{64}^{46}, \cdots, \delta_{64}^{48}, \delta_{64}^{54}, \cdots, \delta_{64}^{56}, \delta_{64}^{62}, \cdots, \delta_{64}^{63}\right\} .
\end{aligned}
$$

Thus, by resorting to Lemma 4, the set of parameter set solutions to the first FRI in (18) is

$$
\begin{aligned}
\Lambda^{1} & =\Lambda^{1,1} \cap \Lambda^{1,2} \cap \Lambda^{1,3} \cap \Lambda^{1,4} \cap \Lambda^{1,5} \cap \Lambda^{1,6} \\
& =\left\{\delta_{64}^{38}, \cdots, \delta_{64}^{40}, \delta_{64}^{46}, \cdots, \delta_{64}^{48}, \delta_{64}^{54}, \delta_{64}^{62}\right\} .
\end{aligned}
$$

Comparing all the parameter set solutions to the first FRI in (18), we obtain the maximum solution to it as

$$
\delta_{64}^{38}=\delta_{8}^{5} \ltimes \delta_{8}^{6} \sim\left[\begin{array}{ll}
0.3 & 0.2
\end{array}\right]^{\top},
$$

and the minimal solutions as

$$
\begin{aligned}
& \delta_{64}^{48}=\delta_{8}^{6} \ltimes \delta_{8}^{8} \sim\left[\begin{array}{ll}
0.2 & 0
\end{array}\right]^{\top}, \\
& \delta_{64}^{62}=\delta_{8}^{8} \ltimes \delta_{8}^{6} \sim\left[\begin{array}{ll}
0 & 0.2
\end{array}\right]^{\top} .
\end{aligned}
$$

Therefore, by (21), the solution set of the first FRI in (18) is

$$
\Omega^{1}=\left[\begin{array}{c}
0.2 \leq x_{1,1} \leq 0.3 \\
0 \leq x_{2,1} \leq 0.2
\end{array}\right] \cup\left[\begin{array}{c}
0 \leq x_{1,1} \leq 0.3 \\
0.2
\end{array}\right] .
$$

Similar to the resolution of the first FRI in (18), all the parameter set solutions to the second and third FRIs in (18) can be successively obtained as

$$
\begin{aligned}
& \Lambda^{2}=\left\{\delta_{64}^{19}, \delta_{64}^{20}, \delta_{64}^{27}, \delta_{64}^{28}, \delta_{64}^{35}, \delta_{64}^{36} \delta_{64}^{43}, \delta_{64}^{44}\right\}, \\
& \Lambda^{3}=\left\{\delta_{64}^{36}, \delta_{64}^{37}, \delta_{64}^{38}, \delta_{64}^{44}, \delta_{64}^{45}\right\} .
\end{aligned}
$$

Then, we can obtain their solution sets as

$$
\begin{aligned}
& \Omega^{2}=\left[\begin{array}{l}
0.2 \leq x_{1,2} \leq 0.5 \\
0.4 \leq x_{2,2} \leq 0.5
\end{array}\right], \\
& \Omega^{3}=\left[\begin{array}{c}
0.3 \\
0.2 \leq x_{2,3} \leq 0.4
\end{array}\right] \cup\left[\begin{array}{l}
0.2 \leq x_{1,3} \leq 0.3 \\
0.3 \leq x_{2,3} \leq 0.4
\end{array}\right] .
\end{aligned}
$$

To sum up, the solution set of (17) is

$$
\begin{aligned}
\Omega= & {\left[\begin{array}{ccc}
0.2 \leq x_{1,1} \leq 0.3 & 0.2 \leq x_{1,2} \leq 0.5 & 0.3 \\
0 \leq x_{2,1} \leq 0.2 & 0.4 \leq x_{2,2} \leq 0.5 & 0.2 \leq x_{2,3} \leq 0.4
\end{array}\right] } \\
& \bigcup\left[\begin{array}{ccc}
0.2 \leq x_{1,1} \leq 0.3 & 0.2 \leq x_{1,2} \leq 0.5 & 0.2 \leq x_{1,3} \leq 0.3 \\
0 \leq x_{2,1} \leq 0.2 & 0.4 \leq x_{2,2} \leq 0.5 & 0.3 \leq x_{2,3} \leq 0.4
\end{array}\right] \\
& \bigcup\left[\begin{array}{ccc}
0 \leq x_{1,1} \leq 0.3 & 0.2 \leq x_{1,2} \leq 0.5 & 0.3 \\
0.2 & 0.4 \leq x_{2,2} \leq 0.5 & 0.2 \leq x_{2,3} \leq 0.4
\end{array}\right] \\
& \bigcup\left[\begin{array}{ccc}
0 \leq x_{1,1} \leq 0.3 & 0.2 \leq x_{1,2} \leq 0.5 & 0.2 \leq x_{1,3} \leq 0.3 \\
0.2 & 0.4 \leq x_{2,2} \leq 0.5 & 0.3 \leq x_{2,3} \leq 0.4
\end{array}\right] .
\end{aligned}
$$


Consider the entry in the first row and second column of $H$. In fact, for any $0<|\varepsilon|<0.1$, we have

$$
0.4<0.5+\varepsilon<0.6
$$

which implies that $\Phi^{1}$ is unchangeable. Thus, the solution set of the first FRI in (18) is unchangeable and the solution set of (17) is unchangeable.

Example 5. Solve the following latticized linear programming:

$$
\begin{gathered}
\min z=\left[\begin{array}{ll}
0.2 & 1
\end{array}\right] \circ\left[\begin{array}{l}
x_{1} \\
x_{2}
\end{array}\right] \\
\text { s.t. }\left[\begin{array}{cc}
0 & 0.1 \\
0.1 & 0 \\
0.3 & 0.1
\end{array}\right] \leq\left[\begin{array}{llll}
0.1 & 0.2 & 0.7 & 0.5 \\
0.4 & 0.2 & 0.3 & 0.1 \\
0.2 & 0.7 & 0.4 & 0.3
\end{array}\right] \ltimes_{\mathcal{B}}\left[\begin{array}{l}
x_{1} \\
x_{2}
\end{array}\right] \leq\left[\begin{array}{cc}
0.3 & 0.4 \\
0.7 & 0.2 \\
1 & 0.4
\end{array}\right] .
\end{gathered}
$$

Step 1: Solve FRI constraint (25).

The equivalent form of (25) is

$\left[\begin{array}{llllll}0 & 0.1 & 0.3 & 0.1 & 0 & 0.1\end{array}\right]^{\top} \leq K^{\prime} \circ\left[\begin{array}{ll}x_{1} & x_{2}\end{array}\right]^{\top} \leq\left[\begin{array}{llllll}0.3 & 0.7 & 1 & 0.4 & 0.2 & 0.4\end{array}\right]^{\top}$,

where $K^{\prime}=\left[\begin{array}{llllll}0.1 & 0.4 & 0.2 & 0.2 & 0.2 & 0.7 \\ 0.7 & 0.3 & 0.4 & 0.5 & 0.1 & 0.3\end{array}\right]^{\top}:=\left(k_{i, j}^{\prime}\right), i=1,2, \cdots, 6, j=1,2$.

The parameter set of (26) is

$$
\Phi=\{0,0.1,0.2,0.3,0.4,0.5,0.7,1\},
$$

and each element in $\Phi$ can be identified by vector form as

$$
0 \sim \delta_{8}^{8}, 0.1 \sim \delta_{8}^{7}, 0.2 \sim \delta_{8}^{6}, 0.3 \sim \delta_{8}^{5}, 0.4 \sim \delta_{8}^{4}, 0.5 \sim \delta_{8}^{3}, 0.7 \sim \delta_{8}^{2}, 1 \sim \delta_{8}^{1} .
$$

Then, the inequalities of (26) can be respectively converted into the following algebraic forms:

$$
\begin{aligned}
& \delta_{8}^{8} \geq M_{1} \ltimes x \geq \delta_{8}^{5}, \delta_{8}^{7} \geq M_{2} \ltimes x \geq \delta_{8}^{2}, \\
& \delta_{8}^{5} \geq M_{3} \ltimes x \geq \delta_{8}^{1}, \delta_{8}^{7} \geq M_{4} \ltimes x \geq \delta_{8}^{4}, \\
& \delta_{8}^{8} \geq M_{5} \ltimes x \geq \delta_{8}^{6}, \delta_{8}^{7} \geq M_{6} \ltimes x \geq \delta_{8}^{4},
\end{aligned}
$$

where

$$
\begin{aligned}
& M_{1}=M_{d}^{8} \ltimes_{t=1}^{2}\left[I_{8^{t-1}} \otimes\left(M_{c}^{8} \ltimes k_{1, t}^{\prime}\right)\right]=\delta_{8}[22345677 \cdots 22345678], \\
& M_{2}=M_{d}^{8} \ltimes_{t=1}^{2}\left[I_{8^{t-1}} \otimes\left(M_{c}^{8} \ltimes k_{2, t}^{\prime}\right)\right]=\delta_{8}[44444444 \cdots 55555678] \text {, } \\
& M_{3}=M_{d}^{8} \ltimes_{t=1}^{2}\left[I_{8^{t-1}} \otimes\left(M_{c}^{8} \ltimes k_{3, t}^{\prime}\right)\right]=\delta_{8}[44445666 \cdots 44445678] \text {, } \\
& M_{4}=M_{d}^{8} \ltimes_{t=1}^{2}\left[I_{8^{t-1}} \otimes\left(M_{c}^{8} \ltimes k_{4, t}^{\prime}\right)\right]=\delta_{8}[33345666 \cdots 33345678] \text {, } \\
& M_{5}=M_{d}^{8} \ltimes_{t=1}^{2}\left[I_{8^{t-1}} \otimes\left(M_{c}^{8} \ltimes k_{5, t}^{\prime}\right)\right]=\delta_{8}[66666666 \cdots 77777778] \text {, } \\
& M_{6}=M_{d}^{8} \ltimes_{t=1}^{2}\left[I_{8^{t-1}} \otimes\left(M_{c}^{8} \ltimes k_{6, t}^{\prime}\right)\right]=\delta_{8}[22222222 \cdots 55555678] \text {, }
\end{aligned}
$$

and $x=x_{1} \ltimes x_{2}$. Thus, one can obtain the set of parameter solutions to (26), denoted by $\Lambda$.

Comparing all the elements in $\Lambda$, we obtain the maximum solution to (26) as

$$
\delta_{64}^{29}=\delta_{8}^{4} \ltimes \delta_{8}^{5} \sim[0.40 .3]^{\top},
$$

and the minimal solution as

$$
\delta_{64}^{61}=\delta_{8}^{8} \ltimes \delta_{8}^{5} \sim\left[\begin{array}{ll}
0 & 0.3
\end{array}\right]^{\top} .
$$


Therefore, according to (21), it is easy to obtain the solution set of FRI (26) as

$$
\Omega=\left[\begin{array}{c}
0 \leq x_{1} \leq 0.4 \\
0.3
\end{array}\right]
$$

Step 2: Calculate the optimal value of the objective function $z$.

The optimal value of $z$ can be calculated as

$$
z_{\min }=\left[\begin{array}{ll}
0.2 & 1
\end{array}\right] \circ\left[\begin{array}{c}
0 \\
0.3
\end{array}\right]=0.3 .
$$

Step 3: Solve the following FRE:

$$
\left[\begin{array}{ll}
0.2 & 1
\end{array}\right] \circ\left[\begin{array}{l}
x_{1} \\
x_{2}
\end{array}\right]=0.3
$$

To obtain all the optimal solutions to (24) and (25), we just need to solve FRE (27) in $\Omega$. The parameter set of (27) is

$$
\Phi^{\prime}=\{0,0.2,0.3,1\}
$$

and each element in $\Phi^{\prime}$ is identified by

$$
0 \sim \delta_{4}^{4}, 0.2 \sim \delta_{4}^{3}, 0.3 \sim \delta_{4}^{2}, 1 \sim \delta_{4}^{1}
$$

Next, we calculate the parameter set solutions to (27).

By Lemma 1, one can obtain the following algebraic form of (27):

$$
M^{\prime} \ltimes x_{1} \ltimes x_{2}=\delta_{4}^{2}
$$

where

$$
M^{\prime}=M_{d}^{4} \ltimes M_{\mathcal{C}}^{4} \ltimes \delta_{4}^{3} \ltimes\left[I_{4} \otimes\left(M_{c}^{k} \ltimes \delta_{4}^{1}\right)\right]=\delta_{4}[1233123312331234] .
$$

Then, by Lemma 4, we can obtain all the parameter set solutions to (27), denoted by $\Lambda^{\prime}$.

Comparing all the elements in $\Lambda^{\prime}$, we find the maximum solution to (27) as

$$
\delta_{16}^{2}=\delta_{4}^{1} \ltimes \delta_{4}^{2} \sim\left[\begin{array}{ll}
1 & 0.3
\end{array}\right]^{\top},
$$

and the minimal solution as

$$
\delta_{16}^{14}=\delta_{4}^{4} \ltimes \delta_{4}^{2} \sim\left[\begin{array}{ll}
0 & 0.3
\end{array}\right]^{\top}
$$

Therefore, according to (21), the solution set of (27) is

$$
\Omega^{\prime}=\left[\begin{array}{c}
0 \leq x_{1} \leq 1 \\
0.3
\end{array}\right]
$$

Step 4: Calculate the optimal solution set of latticized linear programming (24) and (25). The optimal solution set of (24) and (25) is

$$
\begin{aligned}
\mathrm{Y} & =\Omega \cap \Omega^{\prime} \\
& =\left[\begin{array}{c}
0 \leq x_{1} \leq 0.4 \\
0.3
\end{array}\right] \cap\left[\begin{array}{c}
0 \leq x_{1} \leq 1 \\
0.3
\end{array}\right] \\
& =\left[\begin{array}{c}
0 \leq x_{1} \leq 0.4 \\
0.3
\end{array}\right] .
\end{aligned}
$$




\section{Conclusions}

In this paper, we have investigated the resolution of a kind of FRIs with the Boolean semi-tensor product composition. By using the column stacking operator, we have obtained the equivalent column stacking form of FRIs with Boolean semi-tensor product composition, which has the form of SFRIs with max-min composition. Based on the semi-tensor product of matrices, we have obtained the solution set of FRIs with Boolean semi-tensor product composition by finding all possible parameter set solutions, and a general procedure has been developed. It should be pointed out that when fuzzy matrices in FRIs are with compatible dimension, the Boolean semi-tensor product composition coincides with max-min composition. As a result, Boolean semi-tensor product composition is a generalization of max-min composition. Although this paper has obtained all the solutions for FRIs with Boolean semi-tensor product composition, the computational complexity may limit the application of the method proposed in this paper.

Fuzzy relation-based fuzzy control is an important research topic [48]. In the future, we will investigate the fuzzy controller design based on the resolution of FRIs with Boolean semi-tensor product composition. In addition, the sensitivity analysis of FRIs with Boolean semi-tensor product composition is another interesting research topic.

Author Contributions: S.W. and H.L. obtained the solution set of a kind of FRIs with the Boolean semi-tensor product composition, and S.W. drafted the paper. All authors have read and agreed to the published version of the manuscript.

Funding: The work was supported by the National Natural Science Foundation of China under grants 62073202 and 61873150, the Young Experts of Taishan Scholar Project under grant tsqn201909076, and the Natural Science Fund for Distinguished Young Scholars of Shandong Province under grant JQ201613.

Institutional Review Board Statement: Not applicable.

Informed Consent Statement: Not applicable.

Data Availability Statement: Not applicable.

Conflicts of Interest: The authors declare no conflict of interest.

\section{References}

1. Ghodousian, A.; Khorram, E. Fuzzy linear optimization in the presence of the fuzzy relation inequality constraints with max-min composition. Inf. Sci. 2008, 178, 501-519. [CrossRef]

2. Nobuhara, H.; Pedrycz, W.; Hirota, K. Fast solving method of fuzzy relational equation and its application to lossy image compression/reconstruction. IEEE Trans. Fuzzy Syst. 2000, 8, 325-334. [CrossRef]

3. Passion, K.; Yurkovich, S. Fuzzy Control; Addision-Wesley: Boston, MA, USA, 2002.

4. Verbruggen, H.; Babuska, R. Fuzzy Logic Control: Advances in Applications; World Scientific: Singapore, 1999.

5. Sanchez, E. Resolution of composite fuzzy relation equations. Inf. Control 1976, 30, 38-48. [CrossRef]

6. Fang, S.; Li, G. Solving fuzzy relation equations with a linear objective function. Fuzzy Sets Syst. 1999, 103, 107-113. [CrossRef]

7. Pedrycz, W. An identification algorithm in fuzzy relation systems. Fuzzy Sets Syst. 1984, 13, 153-167. [CrossRef]

8. Shieh, B. Solutions of fuzzy relation equations based on continuous t-norms. Inf. Sci. 2007, 177, 4208-4215. [CrossRef]

9. Bourke, M.; Fisher, D. Solution algorithm for fuzzy relational equations with max-product composition. Fuzzy Sets Syst. 1998, 94, 61-69. [CrossRef]

10. Li, J.; Hu, G. An algorithm for fuzzy relation equations with max-product composition. Adv. Fuzzy Sets Syst. 2009, 4, 1-21.

11. Loetamonphong, J.; Fang, S. An efficient solution procedure for fuzzy relation equations with max-product composition. IEEE Trans. Fuzzy Syst. 1999, 7, 441-445. [CrossRef]

12. Cornejo, M.; Lobo, D.; Medina, J. On the solvability of bipolar max-product fuzzy relation equations with the product negation. J. Comput. Appl. Math. 2018, 354, 520-532. [CrossRef]

13. Cornejo, M.; Lobo, D.; Medina, J. On the solvability of bipolar max-product fuzzy relation equations with the standard negation. Fuzzy Sets Syst. 2021, 410, 1-18. [CrossRef]

14. Waziri, M.; Aisha, H.; Mamat, M. A Newton's-like method with extra updating strategy for solving singular fuzzy nonlinear equations. Appl. Math. Sci. 2014, 8, 7047-7057. [CrossRef]

15. Waziri, M.; Majid, Z. A new approach for solving dual fuzzy nonlinear equations using Broyden's and Newton's methods. Adv. Fuzzy Syst. 2012, 2012, 682087. [CrossRef] 
16. Waziri, M.; Moyi, A. An alternative approach for solving dual fuzzy nonlinear equations. Int. J. Fuzzy Syst. 2016, $18,103-107$. [CrossRef]

17. Molai, A.; Khorram, E. An algorithm for solving fuzzy relation equations with max-T composition operator. Inf. Sci. 2008, 178, 1293-1308. [CrossRef]

18. Ignjatovic, J.; Ciric, M.; Damljanovic, N.; Jancic, I. Weakly linear systems of fuzzy relation inequalities: The heterogeneous case. Fuzzy Sets Syst. 2012, 199, 64-91. [CrossRef]

19. Li, P.; Fang, S. On the unique solvability of fuzzy relational equations. Fuzzy Optim. Decis. Mak. 2011, 10, 115-124. [CrossRef]

20. Yeh, C. On the minimal solutions of max-min fuzzy relational equations. Fuzzy Sets Syst. 2008, 159, 23-39. [CrossRef]

21. Perfilieva, I. Fuzzy function as an approximate solution to a system of fuzzy relation equations. Fuzzy Sets Syst. 2004, 147, 363-383. [CrossRef]

22. Perfilieva, I.; Noskova, L. System of fuzzy relation equations with inf- $\rightarrow$ composition: Complete set of solutions. Fuzzy Sets Syst. 2008, 159, 2256-2271. [CrossRef]

23. Cheng, D.; Qi, H. Analysis and Control of Boolean Networks: A Semi-Tensor Product Approach; Springer: London, UK, 2011.

24. Li, H.; Wang, S.; Li, X.; Zhao, G. Perturbation analysis for controllability of logical control networks. SIAM J. Control Optim. 2020, 58, 3632-3657. [CrossRef]

25. Meng, M.; Xiao, G.; Zhai, C.; Li, G. Controllability of Markovian jump Boolean control networks. Automatica 2019, 106, 70-76. [CrossRef]

26. Zhang, K.; Johansson, K. Efficient verification of observability and reconstructibility for large Boolean control networks with special structures. IEEE Trans. Autom. Control 2020, 65, 5144-5158. [CrossRef]

27. Guo, Y.; Li, Z.; Liu, Y.; Gui, W. Asymptotical stability and stabilization of continuous-time probabilistic logic networks. IEEE Trans. Autom. Control 2021. [CrossRef]

28. Li, F.; Tang, Y. Set stabilization for switched Boolean control networks. Automatica 2017, 78, 223-230. [CrossRef]

29. Wang, L.; Liu, Y.; Wu, Z.; Lu, J.; Yu, L. Stabilization and finite-time stabilization of probabilistic Boolean control networks. IEEE Trans. Syst. Man Cybern. Syst. 2021, 51, 1559-1566. [CrossRef]

30. Wu, Y.; Sun, X.; Zhao, X.; Shen, T. Optimal control of Boolean control networks with average cost: A policy iteration approach. Automatica 2019, 100, 378-387. [CrossRef]

31. Chen, H.; Liang, J. Local synchronization of interconnected Boolean networks with stochastic disturbances. IEEE Trans. Neural Netw. Learn. Syst. 2020, 31, 452-463. [CrossRef]

32. Cheng, D.; He, F.; Qi, H.; Xu, T. Modeling, analysis and control of networked evolutionary games. IEEE Trans. Autom. Control 2015, 60, 2402-2415. [CrossRef]

33. Li, C.; Xing, Y.; He, F.; Cheng, D. A strategic learning algorithm for state-based games. Automatica 2020, 113, 108615. [CrossRef]

34. Liu, T.; Wang, J.; Zhang, X.; Cheng, D. Game theoretic control of multiagent systems. SIAM J. Control Optim. 2019, 57, 1691-1709. [CrossRef]

35. Liu, X.; Zhu, J. On potential equations of finite games. Automatica 2016, 68, 245-253. [CrossRef]

36. Zhu, B.; Xia, X.; Wu, Z. Evolutionary game theoretic demand-side management and control for a class of networked smart grid. Automatica 2016, 70, 94-100. [CrossRef]

37. Li, R.; Chu, T.; Wang, X. Bisimulations of Boolean Control Networks. SIAM J. Control Optim. 2018, 56, 388-416. [CrossRef]

38. Zhong, J.; Yu, Z.; Li, Y.; Lu, J. State estimation for probabilistic Boolean networks via outputs observation. IEEE Trans. Neural Netw. Learn. Syst. 2021. [CrossRef]

39. Lu, J.; Li, H.; Liu, Y.; Li, F. A survey on semi-tensor product method with its applications in logical networks and other finite-valued systems. IET Control Theory Appl. 2017, 11, 2040-2047. [CrossRef]

40. Lyu, H.; Wang, W.; Liu, X.; Wang, Z. Modeling of multivariable fuzzy systems by semitensor product. IEEE Trans. Fuzzy Syst. 2020, 28, 228-235. [CrossRef]

41. Lyu, H.; Wang, W.; Liu, X. Universal approximation of fuzzy relation models by semitensor product. IEEE Trans. Fuzzy Syst. 2020, 28, 2972-2981. [CrossRef]

42. Fan, H.; Feng, J.; Meng, M. Solutions to fuzzy relation inequality $A \circ X \circ B \leq$ C. Control Theory Appl. 2016, 33, 694-700.

43. Yan, Y.; Chen, Z.; Liu, Z. Solving type-2 fuzzy relation equations via semi-tensor product of matrices. Control Theory Technol. 2014, 12, 173-186. [CrossRef]

44. Cheng, D.; Feng, J.; Lv, H. Solving fuzzy relational equations via semi-tensor product. IEEE Trans. Fuzzy Syst. 2012, 20, 390-396. [CrossRef]

45. Li, H.; Wang, Y. A matrix approach to latticized linear programming with fuzzy-relation inequality constraints. IEEE Trans. Fuzzy Syst. 2013, 21, 781-788. [CrossRef]

46. Wang, S.; Li, H. Column stacking approach to resolution of systems of fuzzy relational inequalities. J. Frankl. Inst. 2019, 356, 3314-3332. [CrossRef]

47. Cheng, D.; Xu, Z.; Shen, T. Equivalence-based model of dimension-varying linear systems. IEEE Trans. Autom. Control 2020, 65, 5444-5449. [CrossRef]

48. Feng, J.; Lv, H.; Cheng, D. Multiple fuzzy relation and its application to coupled fuzzy control. Asian J. Control 2013, 15, 1313-1324. [CrossRef] 